\title{
CT evaluation of placental abruption in pregnant trauma patients
}

\author{
Sindy H. Wei • Mohammad Helmy • Allen J. Cohen
}

Received: 1 December 2008 / Accepted: 24 February 2009/Published online: 11 March 2009

(C) The Author(s) 2009. This article is published with open access at Springerlink.com

\begin{abstract}
The purpose of the study was to assess the possibility of placental injury detection on computed tomography $(\mathrm{CT})$ in pregnant trauma patients. The images and dictated reports of $44 \mathrm{CT}$ scans of pregnant women who presented to the University of California Irvine Medical Center (UCIMC) from 2003 to 2008 for traumatic abdominal conditions were reviewed for placental abruption. Performances of original dictated reports, an untrained reviewer, and a trained reviewer (who was trained on 22 non-traumatic scans) were compared. Of the 66 pregnant women who received abdominal CT scans, 44 sustained abdominal trauma. Seven suffered placental abruptions, all of which were identified on CT. Sensitivity and specificity were $100 \%$ and $79.5 \%$, respectively, for the untrained reviewer, $100 \%$ and $82.1 \%$ for the trained reviewer, and $42.9 \%$ and $89.7 \%$ for the original dictated reports. Placental abruptions are often overlooked on CT scan. Sensitivity may be improved by systematic evaluation of the placenta and specificity by training on normal placental morphology.
\end{abstract}

All authors on this abstract have nothing to disclose in relation to this activity.

This study was approved by our IRB on 11/30/2007 (HS\# 2007-6023).

S. H. Wei

Department of Medicine,

University of California Irvine Medical Center,

101 The City Drive,

Orange, CA 92868, USA

M. Helmy • A. J. Cohen $(\bowtie)$

Department of Radiological Sciences,

University of California, Irvine Medical Center,

101 The City Drive,

Orange, CA 92868, USA

e-mail: ajcohen@uci.edu
Keywords Placental abruption · Pregnancy · Computed tomography $\cdot$ Trauma $\cdot$ Fetus $\cdot$ Uterus
Abbreviations
CT computed tomography
US ultrasonography
GA gestational age

\section{Introduction}

Traumatic injuries affect $6-7 \%$ of all pregnancies and may necessitate radiographic evaluation for maternal-fetal complications such as placental abruption and preterm labor $[1,2]$. This is often accomplished by computed tomography (CT) scanning of the abdomen and pelvis in the emergent setting. However, the utility of this modality during pregnancy has been limited due to concerns for teratogenicity and childhood cancers caused by fetal irradiation, which are thought to be most detrimental in early pregnancy $[3,4]$. As a result, there have been few CT studies of normal and abnormal placental anatomy $[5,6]$, which in turn may lead to unsatisfactory evaluation of placental abnormalities.

When a timely and sensitive diagnosis affects management and outweighs the risks of radiation exposure, CT scans may be performed during pregnancy [7] and is invaluable in the evaluation of the abdomen, including the uterus, retroperitoneal space, and the fetus after trauma $[8,9]$. With low-dose radiation protocols and improved technology, CT scanning has become an acceptable method for providing fast, comprehensive surveys. As the number of CT studies being performed on pregnant trauma patients increases, so does the need to delineate what constitutes 
normal variation and abnormal appearance on CT scans. Because the normal gravid uterus and physiological changes during pregnancy often confound the interpretation of CT studies, it is also useful to define the anatomical variations associated with normal placental development [9].

This is a case-review study of abdominal and pelvic CT scans of 66 pregnant patients who presented to the UCIMC for abdominal complaints or trauma. We hypothesize that placental abruptions are easily identifiable but often remain undetected on CT scan. Our aims are two-fold: (1) First, we compared reviewer sensitivity and specificity in identifying placental abruption on trauma CT scans. (2) Given the scarcity of information on placental abruptions diagnosed by $\mathrm{CT}$, we characterized the appearance of the maturing placenta through each trimester using non-trauma CT scans and identified the features of placental abruption, which could serve as guides for better detection.

\section{Materials and methods}

This case-review study was approved by the institutional review board. Informed consent was waived. Review of medical records was done in compliance with Health Insurance Portability \& Accountability Act guidelines.

We reviewed 73 consecutive CT scans of both abdomen and pelvis from pregnant women who presented to the UCIMC from March of 2003 to May of 2008 with abdominal complaints. CT studies without IV contrast $(n=$ 6 ) and those studies limited by large body habitus $(n=1)$ were excluded from our analysis. The remaining 66 scans served as the database for our study. These scans were performed for the evaluation of non-traumatic abdominal complaints $(n=22)$ or of trauma associated with onset of abdominal pain $(n=44)$. The majority of the trauma patients suffered from non-penetrating blunt injuries resulting from motor vehicle accidents. There were also a small number of automobile versus pedestrian, falls, and one penetrating gunshot wound. All the non-trauma CT studies $(n=22)$ utilized both oral and IV contrast, whereas $60 \%(n=27)$ of trauma studies used both oral and IV contrast and $40 \%$ $(n=18)$ used IV contrast alone [10]. In the trauma group, one patient who presented for trauma returned 9 days later for continuing symptoms and received a repeat scan. Another trauma patient was pregnant with twins. Therefore, there were a total of 44 trauma patients who received $45 \mathrm{CT}$ scans, resulting in the evaluation of 46 placentas.

First, we reviewed the medical records (including laboratory results, discharge summaries, operative reports, and ultrasonography (US) reports) from each patient whose scans were included in the study to verify the presence or absence of placental pathology. We then reviewed the scans, which were negative for abruption to determine normal variability in appearance on CT scan of the placenta during each trimester of pregnancy. For our purposes, the first trimester was defined as the period of time from conception to the end of 13 weeks of gestational age (GA), the second trimester as between the start of 14 weeks of GA to the end of 26 weeks of GA, and the third trimester as greater than 27 weeks of GA.

A senior reviewer from the Department of Radiological Sciences (AJC), who had previously interpreted some of these studies initially, was asked to review all non-trauma and trauma scans in this study for the targeted purpose of identifying placental abruption. The senior reviewer was not informed of the discharge diagnosis and served as the "untrained" reviewer. A second independent reviewer $(\mathrm{MH})$ was first "trained" on the non-trauma CT scans (all negative for abruption) and subsequently asked to classify the appearance of the placenta as being positive or negative for abruption on all the trauma CT scans. The accuracy of the untrained reviewer, trained reviewer, and original dictated reports in identifying placental abruption were compared in order to isolate the potential difficulties in CT evaluation of placental injuries after trauma.

\section{Results}

\section{Clinical data}

Our series comprised of 66 patients, divided into 22 nontrauma and 44 trauma cases. The average age at presentation was 24.9 years (range, 17-37) in non-trauma patients

Table 1 Clinical data

\begin{tabular}{lll}
\hline & Non-trauma & Trauma \\
\hline $\begin{array}{l}\text { Number of patients } \\
\text { Age of patients }\end{array}$ & 22 & 44 \\
Average \pm standard deviation & $24.9 \pm 5.2$ & $27.5 \pm 6.6$ \\
Range & $17-37$ & $17-43$ \\
Gestational age (weeks) & & \\
Average \pm standard deviation & $26.4 \pm 6.1$ & $23.1 \pm 9.1$ \\
Range & $12-36$ & $5-37$ \\
Number of CT studies & & \\
All trimesters & 22 & 45 \\
1st trimester & $1(4.5 \%)$ & $7(15.6 \%)$ \\
2nd trimester & $8(36.4 \%)$ & $20(44.4 \%)$ \\
3rd trimester & $13(59.1 \%)$ & $18(40.0 \%)$ \\
Number of placentas evaluated & 22 & 46 \\
Number of abruptions & 0 & 7 \\
Rate of abruptions & $0 \%$ & $15.2 \%$ \\
\hline
\end{tabular}


Table 2 Reviewer performance for the identification of placental abruption on CT scans of trauma patients

\begin{tabular}{|c|c|c|c|c|c|c|c|}
\hline & \multicolumn{2}{|c|}{ Negative for abruption } & \multicolumn{2}{|c|}{ Positive for abruption } & \multirow[t]{2}{*}{ DR vs. AJC } & \multirow[t]{2}{*}{ DR vs. MH } & \multirow[t]{2}{*}{ AJC vs. MH } \\
\hline & $\mathrm{TN}$ & $\mathrm{FP}$ & $\mathrm{TP}$ & $\mathrm{FN}$ & & & \\
\hline \multicolumn{8}{|c|}{ Original dictated reports (DR) } \\
\hline 1st trimester & 6 & 0 & 0 & 0 & & & \\
\hline 2nd trimester & 15 & 2 & 1 & 3 & & & \\
\hline 3rd trimester & 14 & 2 & 2 & 1 & & & \\
\hline All GA & 35 & 4 & 3 & 4 & & & \\
\hline Percent all GA & 76.1 & 8.7 & 6.5 & 8.7 & & & \\
\hline \multicolumn{8}{|c|}{ Untrained reviewer (AJC) } \\
\hline 1st trimester & 6 & 0 & 1 & 0 & & & \\
\hline 2nd trimester & 11 & 6 & 3 & 0 & & & \\
\hline 3rd trimester & 14 & 2 & 3 & 0 & & & \\
\hline All GA & 31 & 8 & 7 & 0 & & & \\
\hline Percent all GA & 67.4 & 17.4 & 15.2 & 0.0 & & & \\
\hline \multicolumn{8}{|l|}{ Trained reviewer $(\mathrm{MH})$} \\
\hline 1st trimester & 6 & 0 & 1 & 0 & & & \\
\hline 2nd trimester & 13 & 4 & 3 & 0 & & & \\
\hline 3rd trimester & 13 & 3 & 3 & 0 & & & \\
\hline All GA & 32 & 7 & 7 & 0 & & & \\
\hline Percent all GA & 69.6 & 15.2 & 15.2 & 0.0 & & & \\
\hline \multicolumn{8}{|l|}{ Inter-reviewer agreement } \\
\hline Percent agreement & & & & & 78.3 & 71.7 & 89.1 \\
\hline Percent disagreement & & & & & 21.7 & 28.3 & 10.9 \\
\hline
\end{tabular}

$T N$ true negative, $F P$ false positive, $T P$ true positive, $F N$ false negative

compared with 27.5 years (range, 17-43) in trauma patients. The average GA in the non-trauma group was 26.4 weeks (range 12-36) compared with 23.1 weeks (range 5-37) in the trauma group. Of the $22 \mathrm{CT}$ studies in the non-trauma group, there were one (4.5\%), eight (36.4\%), and 13 (59.1\%) done in the first, second, and third trimesters, respectively. Of the $45 \mathrm{CT}$ studies in the trauma group (one patient came back for repeat imaging one week after presentation), there were seven (15.6\%), $20(44.4 \%)$, and $18(40.0 \%)$ done in the first, second, and third trimesters, respectively. There were no confirmed placental abruptions in the non-trauma series, compared with seven abruptions $(15.2 \%)$ in the trauma series. For a summary of clinical data, please refer to Table 1 .

\section{Reviewer performance}

Of the 46 trauma scans, there were seven placental abruptions. Both the senior reviewer and independent trained reviewer were able to identify all placental abruptions in the trauma series, resulting in sensitivity of $100 \%$ for both reviewers. The reviewer who was trained on the normal scans had one less false positive $(n=7$ of 39 , specificity $=82.1 \%)$ than the untrained reviewer $(n=8$ of 39 , specificity $=79.5 \%$ ). In comparison, the original dictated reports had even fewer false positives ( $n=4$ of 39 , or $10.3 \%$ ) but only identified three of the seven abruptions, resulting in a sensitivity of $42.9 \%$ and a specificity of $89.7 \%$. Of the four false negative original dictated reports, two made no mention
Table 3 Statistical analysis of reviewer performance in identifying placental abruptions

$P P V$ positive predictive value, $N P V$ negative predictive value

\begin{tabular}{lccc}
\hline & Original dictated reports (\%) & Untrained (\%) & Trained (\%) \\
\hline Sensitivity & 42.9 & 100.0 & 100.0 \\
Specificity & 89.7 & 79.5 & 82.1 \\
PPV & 42.9 & 46.7 & 50.0 \\
NPV & 89.7 & 100.0 & 100.0 \\
Accuracy & 82.6 & 82.6 & 84.8 \\
\hline
\end{tabular}



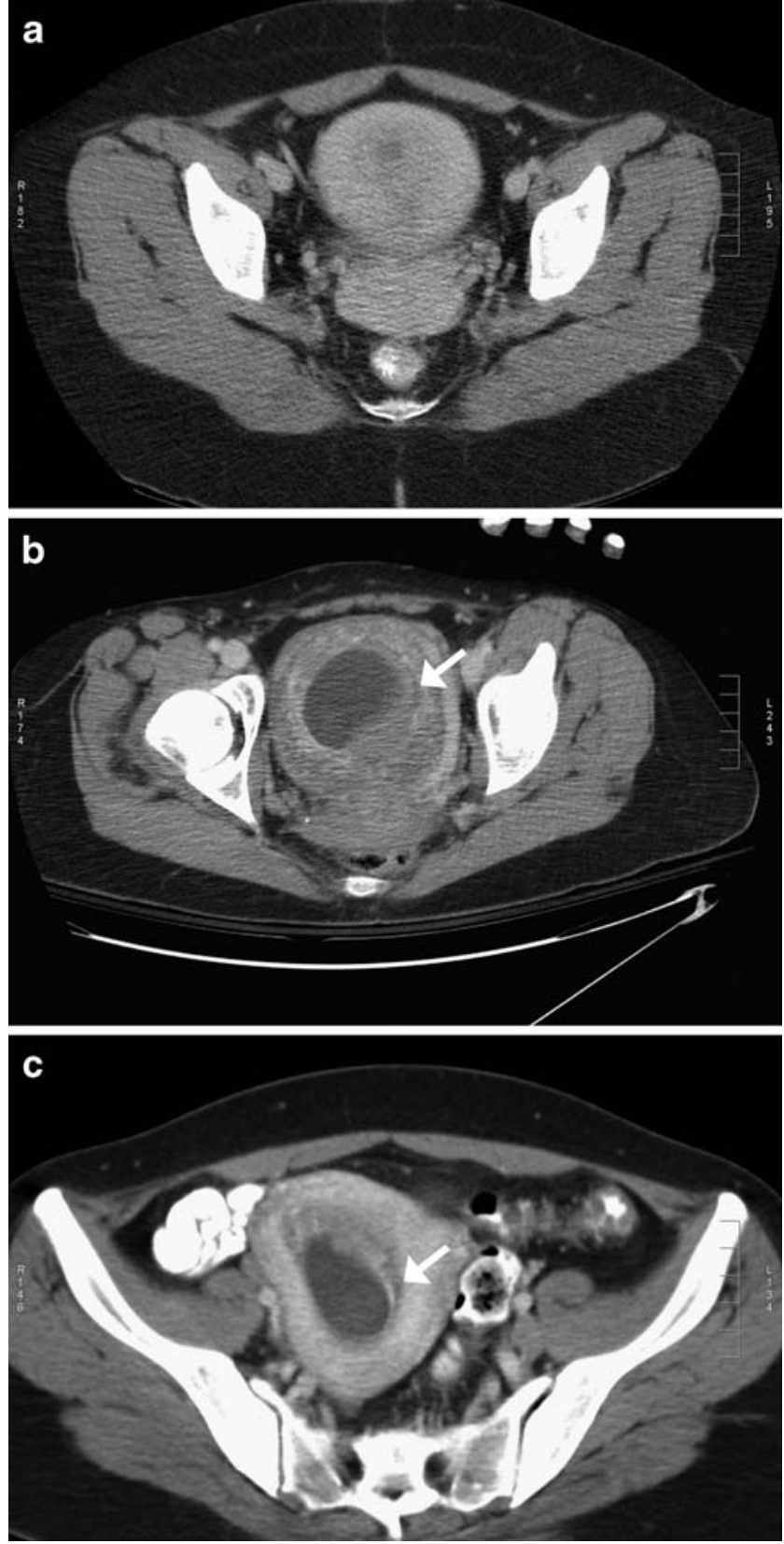

Fig. 1 Normal placentation during the first trimester. a At 5 weeks of GA, the placenta is not distinguished from uterine components, and the fetus is not visible. Subchorionic fluid collections (white arrows) seen at b 10 weeks and at c 12 weeks. CT scans at 5 and 10 weeks were negative for placental injury and taken from the trauma series. The CT scan at 12 weeks was taken from the non-trauma series

of placental appearance at all. See Tables 2 and 3 for summary and statistics (sensitivity, specificity, PPV, NPV, Accuracy) of reviewer performance.

We next present our findings of normal and abnormal placental anatomy on our series of CT scans in order to demonstrate the critical reasons for missed abruptions and false positives.
Normal placental anatomy

During the first trimester, the developing placenta has a relatively homogeneous appearance with a smooth chorionic plate but is not clearly distinguishable from myometrium until late in the trimester (Fig. 1a-c). During the process of placentation, subchorionic hemorrhages may form as a part of early placental development, often without clinical significance (Fig. 1b, c). In contrast, a scan that was associated with spontaneous abortion exactly 1 week after the traumatic event appeared to have increased heterogeneity and a larger subchorionic fluid collection when compared to the normal scans (Fig. 2). During progression into the second trimester, the placenta takes on increased heterogeneity of appearance (Fig. 3a-c). In particular, myometrial contractions are more frequently seen (Fig. 3b) and placental cotyledons are well defined towards the end of the trimester (Fig. 3c). Contrast enhancement of the placenta becomes increasingly heterogeneous as maturation continues into the third trimester, with increased visualization of chorionic plate indentations on the fetal side (Fig 4a) and venous lakes on the maternal side of the placenta (Fig. 4b).

Myometrial contraction can often be misinterpreted as placental abruption

In our study, the false positive rate was as high as $20.5 \%$ when reviewers were directed to look for placental abruption. In a few instances, myometrial contractions were wrongly identified as placental injury as the bulging myometrium may be interpreted as a large area of poorly perfused placental tissue (Fig. 5a, b). However, myometrial contractions may be distinguished from placental injury

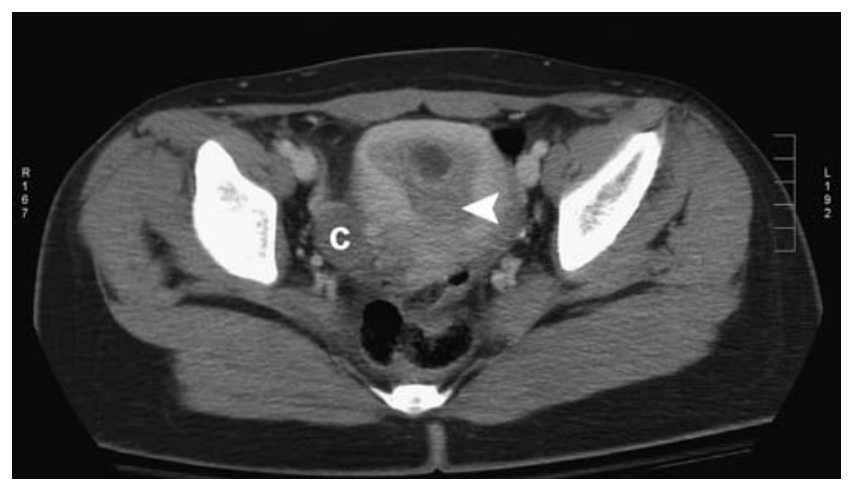

Fig. 2 Questionable placentation during the first trimester. A large collection of subchorionic fluid is seen on this trauma CT study in the mid-first trimester. Seven days after this CT scan was taken, the patient experienced vaginal bleeding, and spontaneous abortion with a collapsed gestational sac was confirmed by US. It is unknown whether the abortion was caused by trauma or preexisting abnormalities. $c$ Incidental finding of a right ovarian cyst 

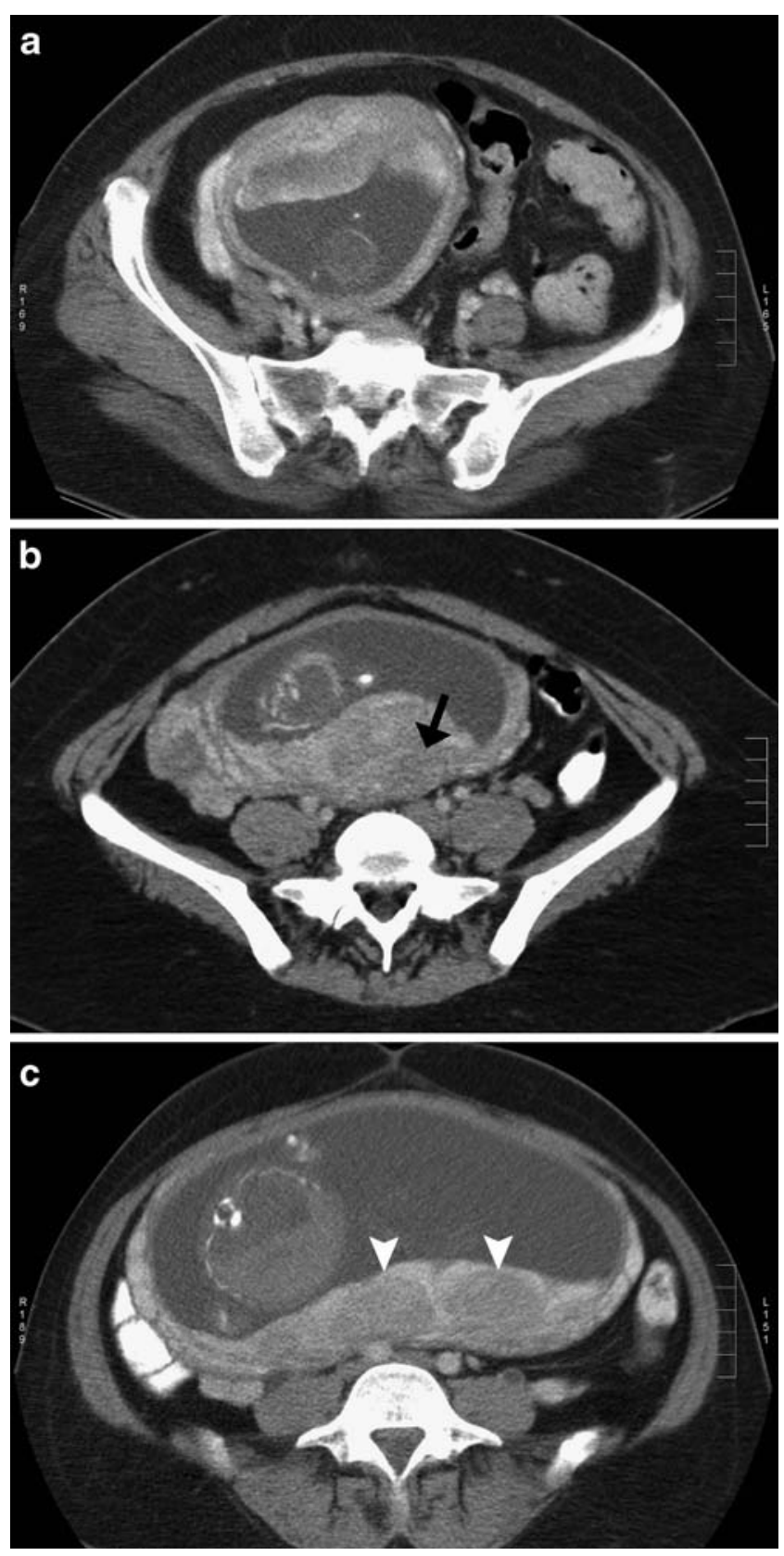

Fig. 3 Normal appearance of the placenta during the second trimester. a At the end of the first trimester ( 13 weeks GA), the placenta is already more heterogeneous and better distinguished from the myometrium than in Fig. 1. b CT scan at 19 weeks shows an increase in the placental-uterine thickness that is caused by contraction of underlying myometrium (black arrow). c By 24 weeks, discrete cotyledons (white arrowheads) can be seen within the placenta. The CT scan at 13 weeks was negative for placental injury and taken from the trauma series. The CT scan at 19 and 24 weeks were taken from the non-trauma series

because they are more likely to form obtuse angles with the myometrium (Fig. 5a, b). Normal variations in placental perfusion may also be interpreted as clinically significant infarcts or placental abruption. Venous lakes contain maternal
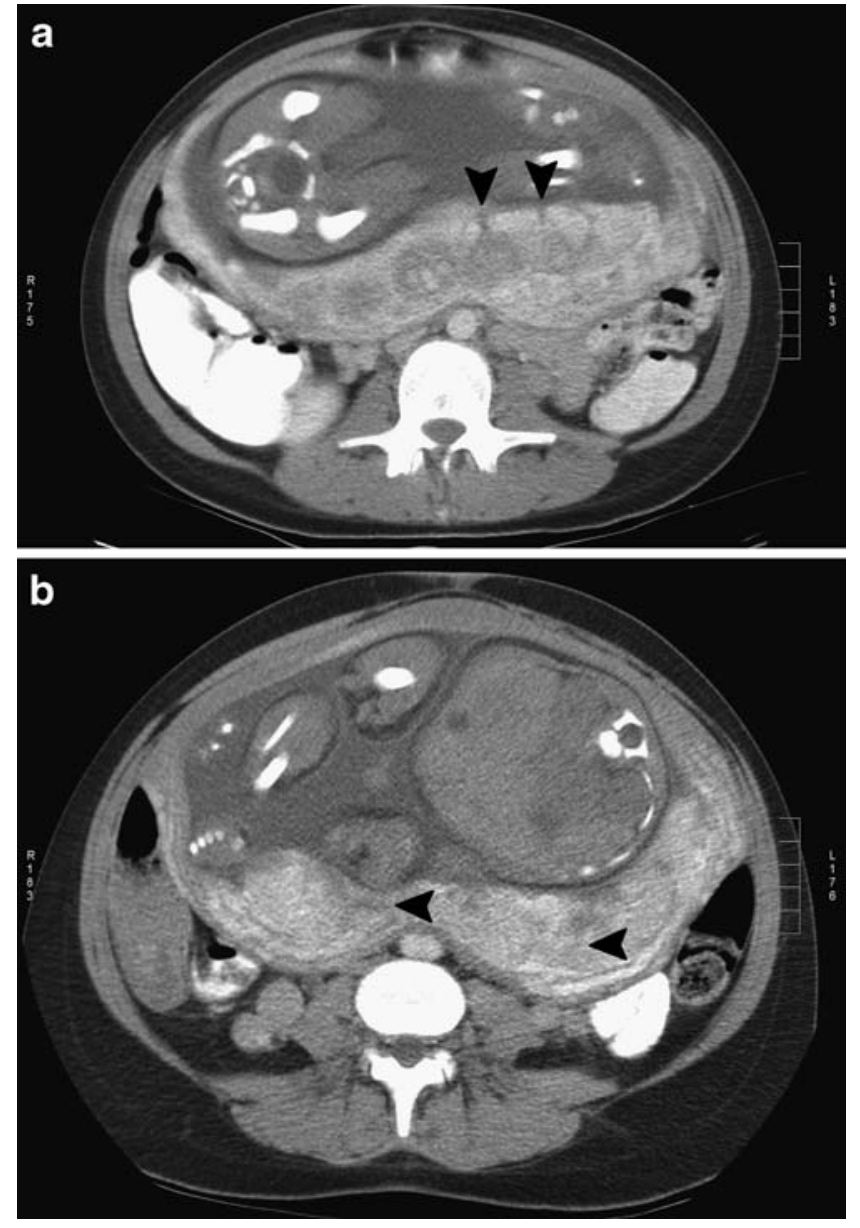

Fig. 4 Normal appearance of the placenta during the third trimester. a Non-traumatic CT scan at 33 weeks of GA demonstrating chorionic plate indentations (black arrowheads). b Non-traumatic CT scan at 36 weeks demonstrating venous lakes (black arrowheads)

blood and are visualized as areas of low enhancement on the maternal side of the placenta. They can be observed in early pregnancy and be mistaken for placental injury (Fig. 5c). Likewise, wedge-shaped placental infarcts increase as the placenta matures (Fig. 5c, d) but are usually of no clinical significance.

Easily identifiable characteristics of placental abruptions on $\mathrm{CT}$

Figure 6 illustrates the $\mathrm{CT}$ appearance of traumatic placental abruptions that were categorized by the original dictated reports as positive for placental abruption. Poorly perfused areas comprised roughly half the total placental area visualized in each cross-sectional image. No obtuse angles were seen between areas of high and low enhancement (Fig. 6, arrows), in contrast to the obtuse angle between nonenhancing myometrial contractions and enhancing placenta (Fig. 5a, b). 

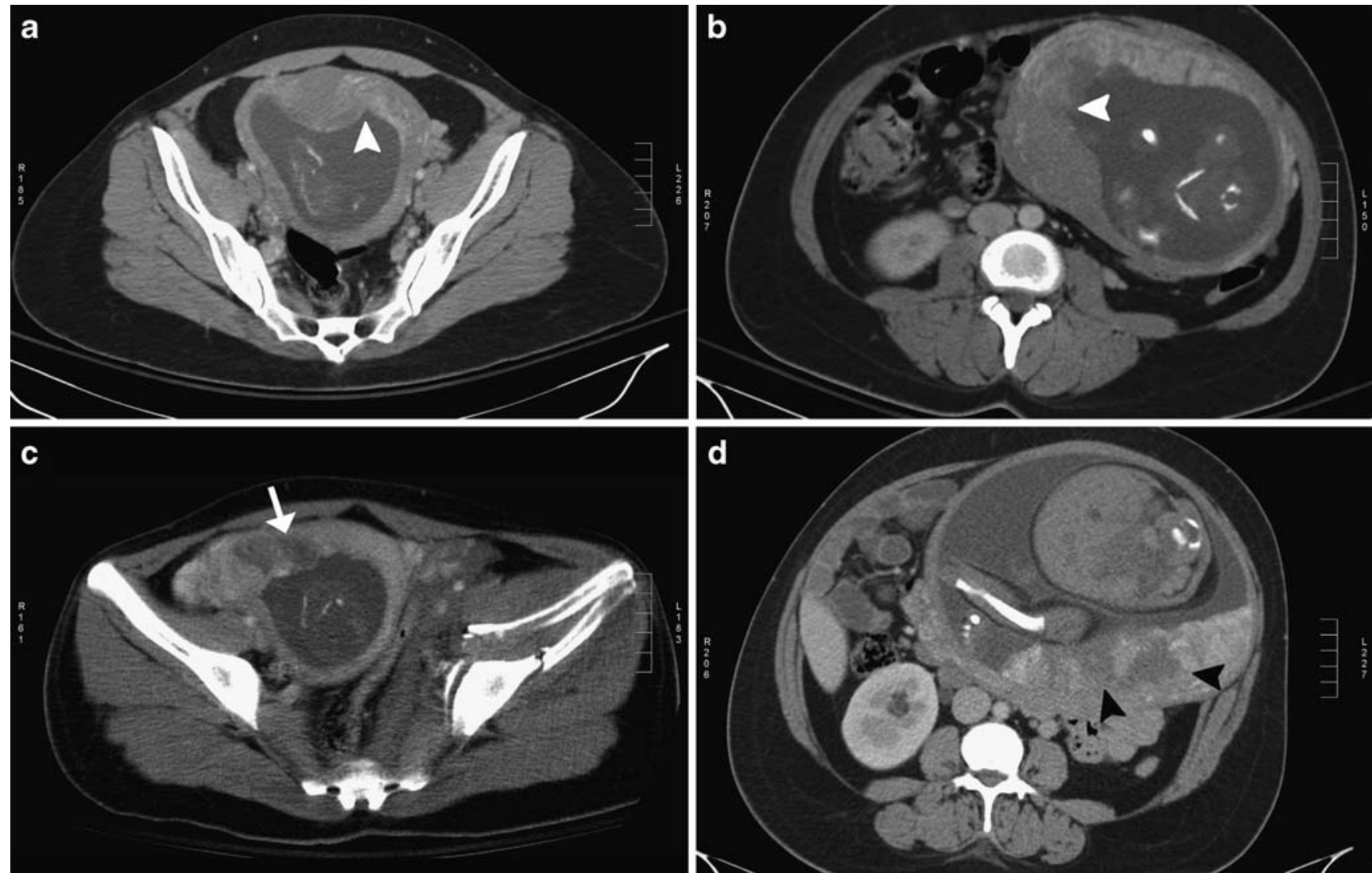

Fig. 5 False positive for placental abruption. CT scans at $\mathbf{a} 14$ and $\mathbf{b}$ 21 weeks, demonstrating myometrial contractions that were clinically insignificant but misinterpreted as positive for abnormal placental pathology. The obtuse angles between the contracting myometrial

bulge and the placenta are marked with white arrowheads. c Venous lakes (white arrow) at 16 weeks. b Clinically insignificant placental infarcts (black arrowheads) at 34 weeks. All of these scans were identified as false positives for placental abruption in the trauma series

Placental abruptions are missed because of lack of placental evaluation

There was a high percentage of false negatives on the original dictated reports (Table 2), which changed clinical management and outcome in at least one case. In two of the dictated reports from the four missed abruptions, placental appearance was not mentioned even though the abnormalities were later readily identified by both the trained and untrained reviewers. One false negative report suggested limited obstetrical US for evaluation of the placenta even though the abnormality strikingly involved a third of the placenta (Fig. 7), and the other reported the placenta as negative for abruption (Fig. 8a).

Large retroplacental hematomas may appear similar to myometrium and be missed

One feature of placental abruption is the development of hematomas that undermine the placental tissue in a retroplacental position (Figs. $7 \mathrm{~b}$ and $8 \mathrm{a}, \mathrm{b}$ ). These should be

differentiated from other types of hematomas found in other locations, e.g., preplacental hematomas of the subchorionic and subamniotic types, marginal hematomas at the lateral placental edge, or physiologic placental infarcts. In two other examinations, placental abruptions with large retroplacental hematomas were missed on the original dictated reports (Fig. 8). The large hematomas have similar intensity compared to the myometrium and therefore can be easily missed even when routine placental evaluation is made for retroplacental bleeding (Fig. 8b).

\section{Discussion}

Placental abruption has an incidence of approximately $1 \%$ in the United States and affects $15-30 \%$ of pregnancies with third trimester bleeding. Complications include preterm labor, fetal distress, fetal death in $20-60 \%$, maternal shock, DIC, and maternal death. It is usually evaluated clinically by maternal symptoms, physical exam, laboratory 

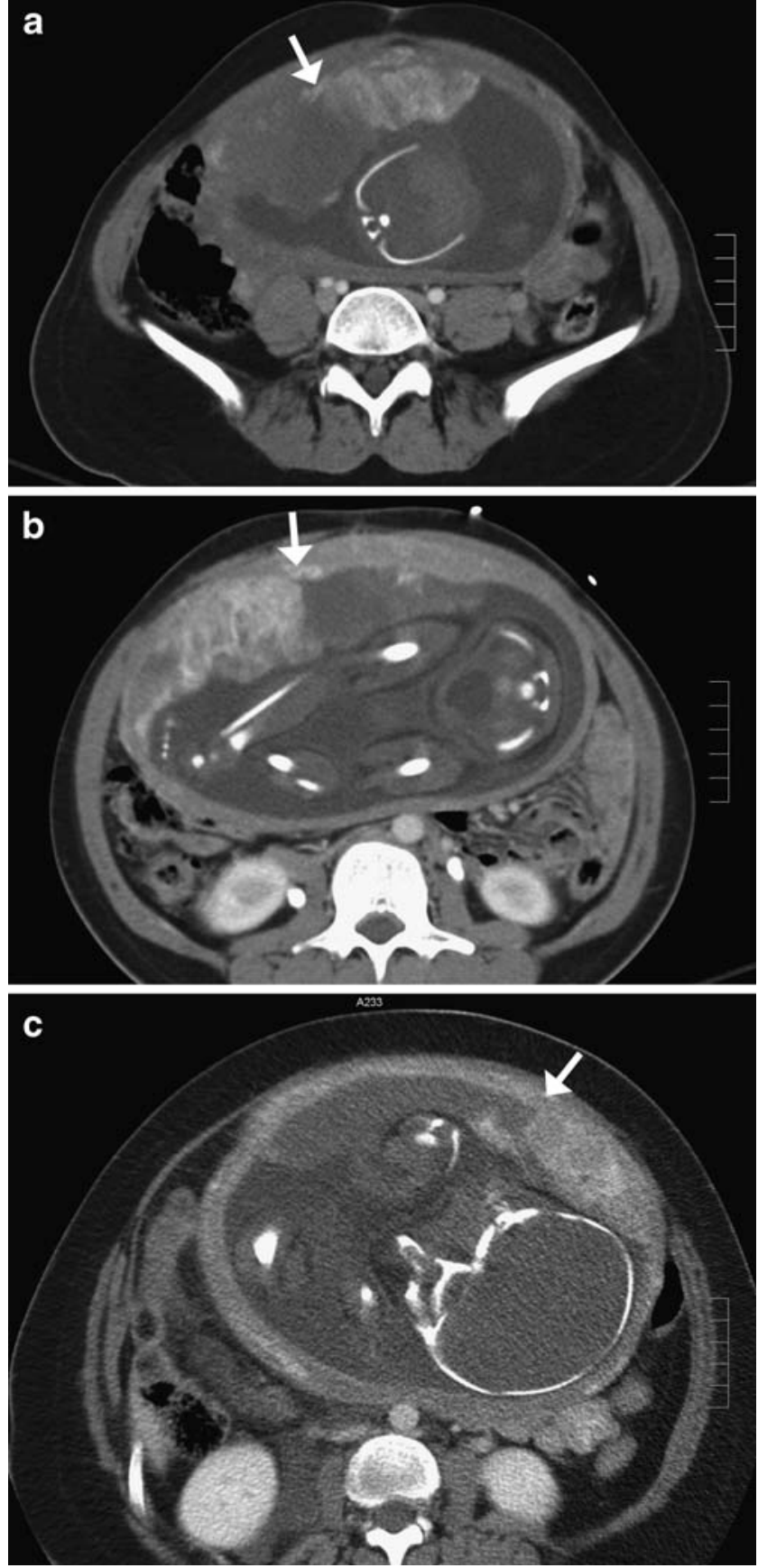

Fig. 6 Placental abruptions correctly identified on CT scan. CT scans taken from the trauma series a at 23 weeks of GA with original dictated report describing a "right-sided placental hematoma", b at 29 weeks of GA with original dictated report describing "absent perfusion in anterior placenta", c at 36 weeks of GA with original dictated report describing "heterogeneous placental enhancement... relative area of decreased enhancement anteriorly, correlate with abruption". White arrows mark the border between non-perfused and perfused regions of the placenta

tests, US, and indirectly by fetal heart rate monitoring if indicated [11]. However, $20 \%$ of hematomas are confined to the uterus and do not manifest as vaginal bleeding. Acute placental abruptions can be seen on US as an echogenic
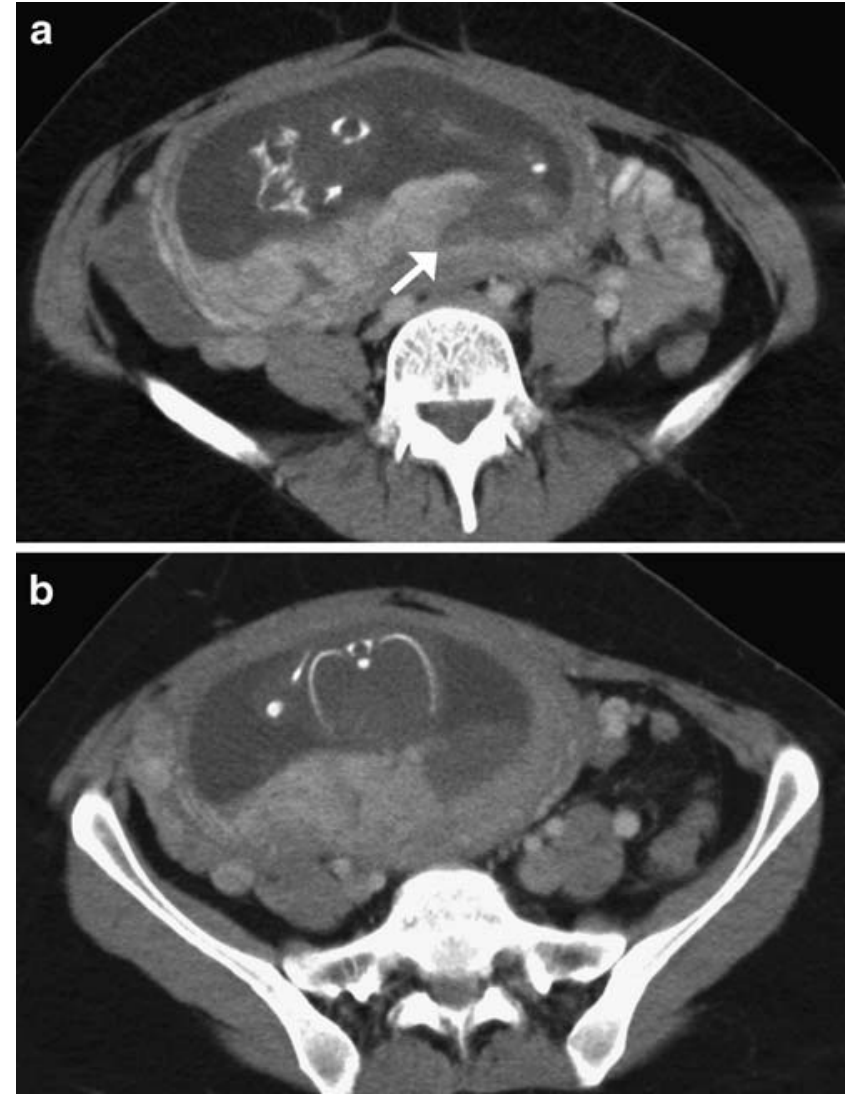

Fig. 7 False negative for abruption: placental abnormality easily identified. Two cross-sectional images from one trauma CT study at 21 weeks of GA demonstrate one example of a missed placental abruption. The original report suggested limited OB U/S to evaluate placenta, but no evaluation of placenta was dictated. a Retroplacental hemorrhage with undermining of the placenta (white arrow). b Nonperfused placenta over a significant area

retroplacental mass, which then become hypoechoic in 12 weeks mimicking fibroids. In contrast to CT scanning, US studies have poor sensitivity for abruption, and the Kleihauer-Betke test has limited value as a diagnostic test [12].

In this study, we reviewed $66 \mathrm{CT}$ scans of pregnant patients who presented to the UCIMC from 2003 to 2008 to study normal and abnormal placental appearance. There is a real need to emphasize the routine evaluation of the placenta in traumatic injuries, especially in the second or third trimesters where management is more likely to be influenced by results of the CT scan. In our hands, routine evaluation of placental abruption increased sensitivity from $42.9 \%$ to $100 \%$ but also lowered specificity by roughly $10 \%$. The trained reviewer achieved a slightly better specificity than the untrained reviewer, suggesting that "training" on the appearance of the normal placenta may serve to decrease the false positive rate. However, more studies would need to be done on the effects of training to confirm its utility. 

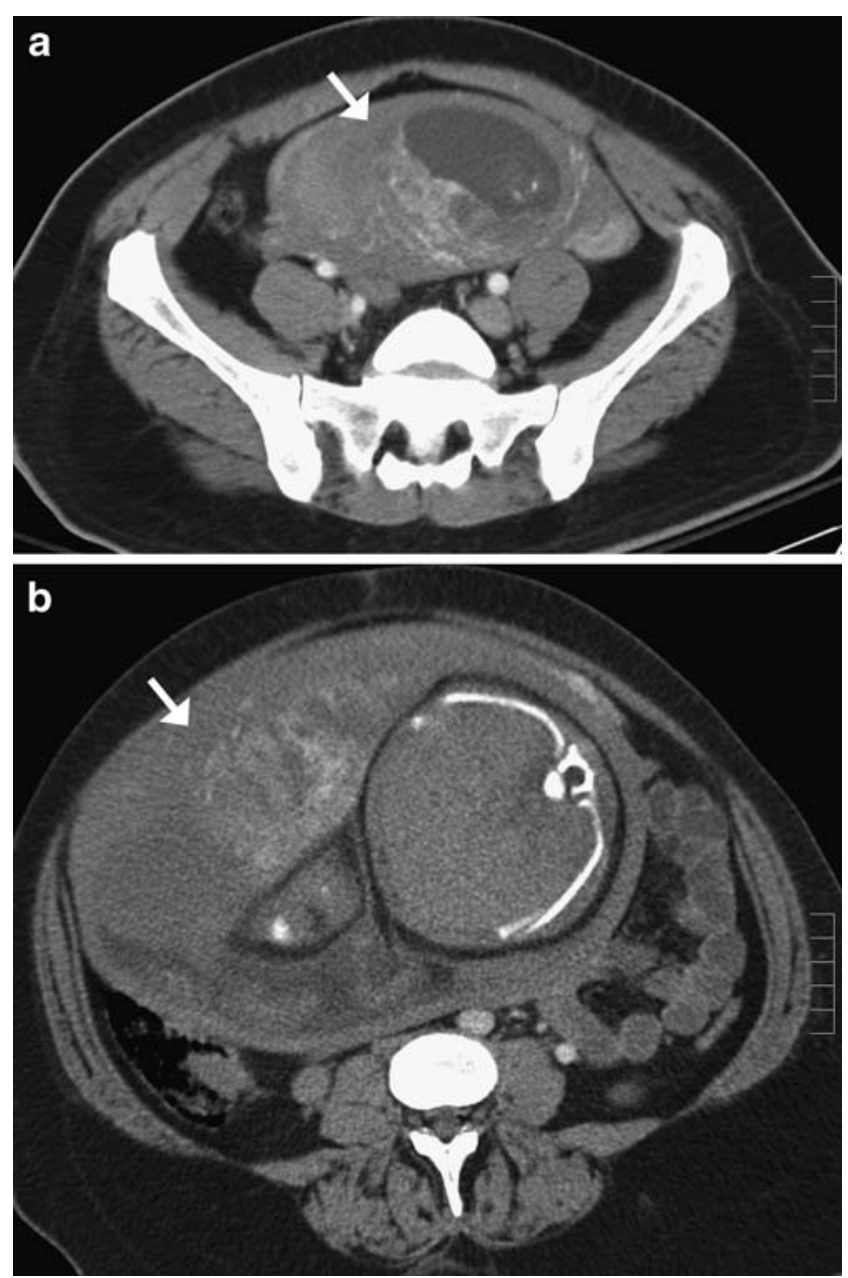

Fig. 8 False negative for placental abruption: large undermining placental hematomas. a CT scan at 13 weeks of GA, with no evaluation of the placenta on the original dictated report. b CT scan in the early third trimester described by original dictated report as "placental anterior... demonstrates heterogeneous enhancement... no subplacental hematoma identified". These CT scans demonstrate large but subtle retroplacental hematomas that developed as a result of traumatic placental abruptions, with undermining of the placenta marked by white arrows

For every one missed abruption in our series, there was one false positive that would have required additional obstetrical monitoring overnight. This is an acceptable cost compared to the catastrophic consequences of missing an abruption, which would also include medical expenses from late complications and psychological burden for the patient. Therefore, in order to achieve a high sensitivity and to improve clinical monitoring and management, it is preferable to have a low threshold of suspicion for placental abruption.

Aberrations in fetal heart rate have been shown to correlate with the severity of placental abruption [11] and may serve as a useful tool to differentiate between false positives and true abruptions diagnosed by CT scan. Recently, imaging of the uteroplacental circulation by Doppler US has been found to be potentially useful in the screening of pregnancies at risk for complications dealing with abnormal placentation [13]. In the future, this technique may possibly be applied in emergency situations to the detection of traumatic abruption, though it is unknown how its performance would compare with CT scanning and how it may improve diagnosis as an adjunct diagnostic imaging modality.

Based on our review of placental appearance on $\mathrm{CT}$, we believe that it is possible to establish criteria for identifying placental abruption. We found that components of normal placentation that appeared as increasing heterogeneity as a function of GA were repeatedly mistaken for abnormalities. These included subchorionic hemorrhages, venous lakes, and wedge-shaped infarcts that were usually limited in size and had no clinical significance. Myometrial contractions mimicked loss of perfusion to placental tissue but were distinguishable by their obtuse angle and lack of undermining of the placenta. Bulky, undermining hematomas from retroplacental abruptions were often missed as they caused the entire placenta to appear more homogenous and lowered detection of perfusion defects.

True placental abruptions were characterized by large, contiguous, and retroplacental and/or full-thickness areas of low enhancement that form acute angles with myometrium. Abruptions involving $>50 \%$ of the placental surface are frequently associated with fetal demise [12]; however, some of these large abruptions remained undetected likely due to the lack of systematic evaluation of the placenta. Many of the original dictated reports for trauma CT scans performed on patients who did not have clinical evidence for abruption also lacked descriptions of the placenta, in spite of the rate of abruption being high enough to warrant routine placental evaluation. This suggests that a lack of routine placental evaluation or the lack of training on the normal and abnormal appearance of the placenta may decrease sensitivity of detecting abruptions. Of the seven placental injuries identified on the 44 trauma CT scans in our study, only three were identified correctly by the original reviewers. Further studies must be done to determine the sensitivity and specificity of detecting placental abruption across institutions. Therefore, we strongly recommend that the evaluation of CT scans in the pregnant patient with abdominal pain-especially in those who suffered abdominal trauma-includes a systematic assessment of the placenta.

Acknowledgments We are grateful to Dr. David Alvarez for his insightful suggestions and the University of California Irvine Medical Scientist Training Program for support of S.H.W. 
Open Access This article is distributed under the terms of the Creative Commons Attribution Noncommercial License which permits any noncommercial use, distribution, and reproduction in any medium, provided the original author(s) and source are credited.

\section{References}

1. El Kady D (2007) Perinatal outcomes of traumatic injuries during pregnancy. Clin Obstet Gynecol 50(3):582-591. doi:10.1097/ GRF.0b013e31811 eab82

2. Weintraub AY, Levy A, Holcberg G et al (2006) The outcome of blunt abdominal trauma preceding birth. Int J Fert Women's Med 51(6):275-279

3. Kusama T, Ota K (2002) Radiological protection for diagnostic examination of pregnant women. Congenit Anom (Kyoto) 42(1):10-14. doi:10.1111/j.1741-4520.2002.tb00848.x

4. Hurwitz LM, Yoshizumi T, Reiman RE et al (2006) Radiation dose to the fetus from body MDCT during early gestation. AJR Am J Roentgenol 186(3):871-876. doi:10.2214/AJR.04.1915

5. Civil ID, Talucci RC, Schwab CW (1988) Placental laceration and fetal death as a result of blunt abdominal trauma. J Trauma 28(5):708-710. doi:10.1097/00005373-198805000-00030
6. Dash N, Lupetin AR (1991) Uterine rupture secondary to trauma: CT findings. J Comput Assist Tomogr 15(2):329-331. doi:10.1097/ 00004728-199103000-00030

7. Barron WM (1984) The pregnant surgical patient: medical evaluation and management. Ann Intern Med 101(5):683-691

8. Ames Castro M, Shipp TD, Castro EE et al (2001) The use of helical computed tomography in pregnancy for the diagnosis of acute appendicitis. Am J Obstet Gynecol 184(5):954-957. doi:10.1067/mob.2001.111721

9. Lowdermilk C, Gavant ML, Qaisi W et al (1999) Screening helical CT for evaluation of blunt traumatic injury in the pregnant patient. Radiographics 19(Spec No):243-255, discussion S256-248

10. Stafford RE, McGonigal MD, Weigelt JA et al (1999) Oral contrast solution and computed tomography for blunt abdominal trauma: a randomized study. Arch Surg 134(6):622-626. doi:10.1001/archsurg.134.6.622. discussion 626-627

11. Usui R, Matsubara S, Ohkuchi A et al (2008) Fetal heart rate pattern reflecting the severity of placental abruption. Arch Gynecol Obstet 277(3):249-253. doi:10.1007/s00404-007-0471-9

12. Oyelese Y, Ananth CV (2006) Placental abruption. Obstet Gynecol 108(4):1005-1016

13. Urban G, Vergani P, Ghidini A et al (2007) State of the art: noninvasive ultrasound assessment of the uteroplacental circulation. Semin Perinatol 31(4):232-239. doi:10.1053/j.semperi.2007.06.002 